\title{
Staphylococcal cutaneous infection in newborn - clinical approach and treatment
}

\author{
Reka Toth ${ }^{1}$, Mihaela Chincesan ${ }^{2,3}$, Ovidiu Grama ${ }^{3,4}$, Septimiu Voidazann ${ }^{5}$, Alina Grama ${ }^{2,3}$ \\ ${ }^{1}$ Department of Quality Management in Healthcare Services, \\ County Emergency Clinical Hospital, Tg. Mures, Romania \\ ${ }^{2}$ Pediatrics Clinic, County Emergency Clinical Hospital, \\ Tg. Mures, Romania \\ ${ }^{3}$ Department of Pediatrics, George Emil Palade University of Medicine, Pharmacy, \\ Sciences and Technology, Tg. Mures, Romania \\ ${ }^{4}$ Obstetrics and Gynecology Clinic, County Emergency Clinical Hospital, Tg. Mures, Romania \\ ${ }^{5}$ Department of Epidemiology, George Emil Palade University of Medicine, Pharmacy, \\ Sciences and Technology, Tg. Mures, Romania
}

\begin{abstract}
Introduction. Staphylococcal scalded skin syndrome (SSSS) is an exfoliating skin disease with two age peak incidence: the neonatal period and early childhood. Differential diagnosis includes bullous impetigo, Stevens-Johnson syndrome, Toxic epidermal necrosis (TEN), oxic shock syndrome, Scarlet fever, Kawasaki disease and Pemphigus.

Case presentation. We present a 14-day-old female, from a rural area, admitted in the Pediatrics Clinic with appearance of blistering skin rash on the face and forehead, extending to the chest, abdomen and limbs, with areas of exfoliation and irritability respectively. The positive diagnosis of SSSS was made based upon the medical history (perforation of the auricular lobules, rapid onset of the vesicles that growth and break), physical findings (multiple vesicles and lax blisters, positive Nikolsky sign, perioral crusts, conjunctivitis with purulent secretions, erythema and bilateral palpebral edema) and paraclinical (lesion secretion - saprophytic flora; conjunctival secretion - Staphylococcus aureus MSSA), and adequate therapeutic response respectively. With parenteral antibiotic treatment, hydro-electrolyte rebalancing and rigorous local hygiene the evolution was favorable.

Conclusions. Staphylococcus aureus is an important pathogen in the neonatal period. The appropriate diagnosis and treatment of infection with hematogenous dissemination in the skin are necessary to reduce morbidity and mortality.
\end{abstract}

Keywords: newborn, staphylococcal scalded skin syndrome (SSSS)

\section{INTRODUCTION}

SSSS is a toxin-mediated skin disorder that primarily affects young children. Preceding terms for SSSS in neonates include Ritter's disease and neonatal pemphigus. The disease occurs when exotoxins (ET) produced by Staphylococcus aureus disseminate hematogenously to the skin. The characteristic clinical findings includes pain and diffuse cutaneous erythema, lax blisters, superficial peeling, superficial erosions, without the involvement of mucous membranes, evidence of concomitant staphylococcal skin or conjunctival infection and positive Nikolsky sign. Associated symptoms may be fever, irritability and poor oral intake. $\mathrm{Pa}-$ tients with this condition usually require hospitalization for administration of parenteral antibiotics 
and rigorous supportive care. The prognosis is very good when properly treated and most patients recover completely without sequelae (1-3).

\section{CASE PRESENTATION}

A 14-day-old female infant is brought to the emergency department with erythematous vesicular rash on the face and folds, which are rapidly expanding to the chest, abdomen and limbs, with areas of peeling manifested 2 days before. Associated she presents irritability, low appetite. Following the assessment of the case it is decided she is to be admitted to the Pediatric Clinic for further investigations and treatment.

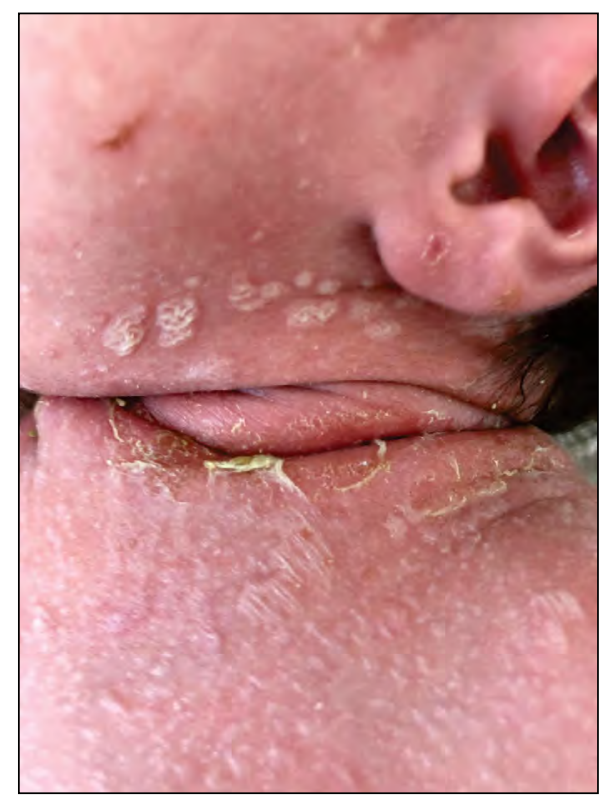

FIGURE 1. Perioral crust

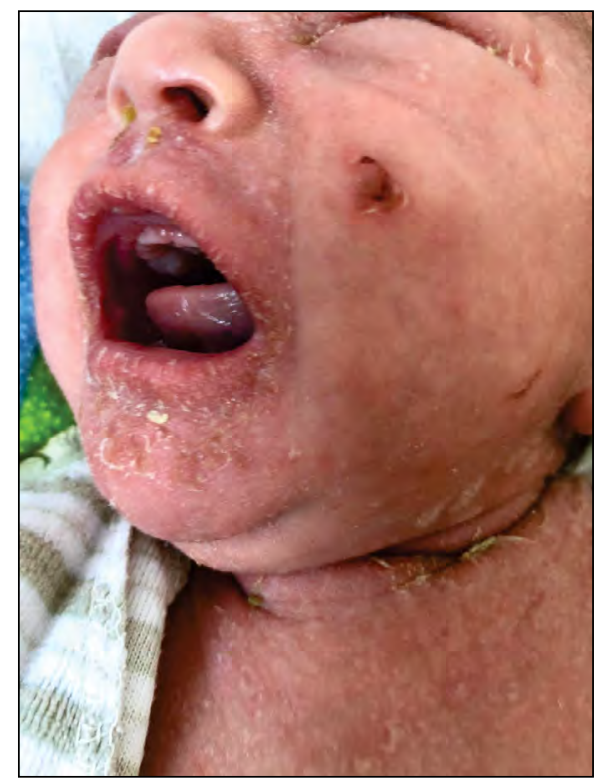

FIGURE 2. Exfoliation in flaps at cervical level
Insignificant family medical history; personal medical history: the second child of the mother, born from an affirmative physiological pregnancy, at term, normal delivery, with BW: 3,030 g, APGAR score: $10 / 1$ ', vaccinated and vitaminized according to age, exclusively naturally fed. From the mothers statements we mention the perforation of the earlobes on the 5th day of life, on the day of maternity discharge, with subsequent local inflammation, for which was recommended local treatment with Tetracycline ointment. It presents purulent conjunctival secretions in the 10th day of life.

Physical examination at admission: influenced general state, afebrile, suffering facies, erythema and bilateral palpebral edema, perioral erythematous crusts with flaking (appearance of dried oatmeal) and around the eyes, $60 \%$ of the body surface covered with blisters and lax bubbles, multiple, of different dimensions with turbid content, spread all over the body, several pustules, positive Nikolsky sign (light mechanical pressure of the skin causes its peeling), with large areas of exfoliation in flaps at the level of the folds - cervical, axillary, inguinal; wrinkled skin, bilateral conjunctivitis with purulent secretions, otherwise normal relations according to age.

Laboratory at presentation: mild anemia (Hgb: $12.6 \mathrm{~g} / \mathrm{dl}$, Htc: $36.10 \%)$, thrombocytosis $(749,000 /$ ul), lympho-monocytosis $(44.6 \%$, respectively $11.3 \%$, negative inflammatory markers, normal renal and hepatic function. The cultures from the skin lesions were negative (bacterial skin flora), respectively conjunctival secretions - positive culture (Staphylococcus aureus MSSA).

Considering the early age, the medical history (superinfected perforation of the earlobes, conjunctivitis, rapid progressive symptoms), SSSS characteristic clinical picture (bladder blisters, with erosive desquamation in folds, facial and later disseminated, positive Nikolsky sign, unaffected mucosa) as provisional diagnosis the SSSS was established and parenteral antibiotic treatment (Oxacillin, Ceftriaxone), analgesics (acetaminophen), hydro-electrolytic rebalancing and rigorous skin hygiene were initiated. Topical treatment (hydrocortisone butyrate, momethasone furoate) was recommended by the dermatologist specialist.

Differential diagnosis of SSSS includes other conditions that present with cutaneous erythema with vesicles: burns - missed in the medical history 
of the disease; bullous impetigo - disseminated vesicles, negative culture at the level of skin lesions, and in Bullous impetigo the lesions appear at the sites of infected skin and the cultures are positive; Stevens-Johnson syndrome and TEN - in our case with no prior drug treatment, without mucosal involvement; Toxic shock syndrome - lack of periorificial crusting and blisters, negative Nikolsky sign distinguished it from SSSS; scarlet fever, respectively Kawasaki disease - perioral crusts, skin fragility and pain observed in SSSS are absent in these conditions; Pemphigus foliaceus and vulgaris are autoimmune bladder disorders that can occur with blisters and erosions similar to SSSS. To confirm the diagnosis, a skin biopsy with direct immunofluorescence microscopy is required. Among the newborns we can also find: epidermolytic ichthyosis, epidermolysis bullousa, bullous mastocytosis, cutaneous candidiasis, ingestion or topical exposure of toxic agents (boric acid, pesticides, household cleaning agents), mercury poisoning, ingestion and excretion of sennoside induces perianal peeling and erythema.

With systemic and locally instituted treatment it presented a favorable evolution, with partial remission of the lesions - these being in different stages of healing.

The medical history of the disease, clinical findings, paraclinical results and the therapeutic response support the final diagnosis of SSSS, without the need for histopathological examination of the skin.

The patient was discharged after 10 days of hospitalization, without sequelaes or pigmentation of the skin with recommendations to respect personal hygiene.

\section{DISCUSSIONS}

SSSS is a dermatological condition caused by epidermolytic ET of some strains of Staphylococcus aureus, the most common subsets belonging to phage group 2 strains 55 and 71 and, the less common causative strains include types $3 \mathrm{~A}, 3 \mathrm{~B}, 3 \mathrm{C}$ and ST121 strains. The two pathogenic toxins produced in SSSS are exfoliative toxin A (ETA) and exfoliative toxin B (ETB) or both at the same time. These toxins induce epidermal vesicles formation by the cleavage of the cell - cell adhesion molecule desmoglein-1, which is only expressed by keratino- cytes in the granular cell layer (4). The condition was first described by Ritter von Rittershain, the SSSS term being defined by Melish and Glasgow. The estimated prevalence ranges from 0.09 to 0.56 cases per million people (3). Staiman A et al. reported a mean annual incidence of 7.67 per million U.S. children, with 45.1 cases per million U.S. infants age $<2$ years, analyzing the national sample of patients in the period of 2008-2012 (5). The disease is most commonly seen in newborns, there are case reports starting with the first two days of life and children younger than 6 years (7-16). The sex ratio is approximately equal in children, and African-American minors are less sensitive than Caucasian children (3). The disease can also occur in older children and adults who have chronic diseases (impaired kidney function, immunodeficiencies). According to reported studies in Hunan (China), it is more frequent in summer and fall, but the revised report from the U.S. do not support seasonal differences, our patient was admitted in October $(5-6,17)$. The onset may be sporadic or outbreaks caused by asymptomatic carriers that spread the disease to sensitive persons, such as newborns in intensive care units or nurseries, our case being sporadic. Paranthaman K et al. reports an outbreak of SSSS with 8 infected newborns at a maternity unit in England, between December 2012 and March 2013, and Pimentel de Araujo F et al. describes an outbreak due to a strain of $S$. aureus producing ETA that occurred in a local hospital in northern Italy between July 2013 and February 2014, when 12 newborns had skin infections, mainly blisters or pustules (18-20). The predisposition of young children to SSSS is due to the lack of protection antibodies against staphylococcal toxins and / or insufficient ability to excrete ET (21).

The disease starts with a local infection, with or without clinical manifestations, due to a Staphylococcus aureus strain that produces exfoliating toxins causing epidermiolysis. The primary source of infection is usually the head and neck region (conjunctivitis, pharyngitis, otitis media), postoperative infection (circumcision), the umbilical area (omphalitis) and the diaper area (pustules, impetigo, cellulitis). In our case, the primary site was conjunctival and appeared two days before the skin lesions. In the medical articles the incubation period ranges from 1 to 10 days (3). 
The clinical features of SSSS comprises a prodromal phase in which the change of general malaise, fever and irritability may be present. The first skin signs appear suddenly: erythroderma, skin pain, skin with a wrinkled appearance on the folds. As the disease progresses, usually within 48 hours, the thin-walled vesicles appear in the erythematous areas, which break easily; superficial peel off in sheets, accentuated in folds (neck, armpits, inguinal, gluteal groove), leaving the skin moist, erythematous, shiny, giving rise to a scalded appearance. Erosion may also occur in friction areas, such as in the perianal region. Gentle pressure on apparently normal skin causes separation of the upper epidermis (positive Nikolsky sign). Facial, periorbital, perioral edema with thick dry crusts and intact mucosae are characteristic of the clinical assessment $(1,3)$.

Post inflammatory hyper- or hypopigmentation occasionally follow healing, especially in patients with hightly pigmented skin and usually disappear within a few months. The scar does not usually appear. Complications of the disease can be secondary infection (cellulite, sepsis, pneumonia), dehydration, electrolyte imbalance and hypothermia (16). In our case without immediate or late complications.

The diagnosis of the disease is usually based on the medical history and clinical findings. Bacterial cultures of sites with suspected staphylococcal infection should be performed to confirm Staphylococcus aureus infection and to provide information on susceptibility to antibiotics. Skin biopsy remains the gold standard although it is usually reserved for cases that do not respond to initial therapy. We can identify the level of epidermal cleavage distinguishing SSSS from other conditions in which cleavage occurs lower in the epidermis or in the subepidermal plane (for example TEN) by biopsy. In our case the onset of the disease, the rapid progression, the bilateral conjunctivitis preceded by the appearance of erythroderma, the cutaneous findings and the paraclinical results, and the therapeutic response respectively, support the diagnosis of SSSS.

SSSS management:

1. eradication of the staphylococcal causative infection

- immediate hospitalization
- intravenous antibiotic therapy - initially penicillinase-resistant penicillin, such as oxacillin or nafcillin, alternatives are $1 \mathrm{st}$ or 2 nd generation cephalosporin or vancomycin; subsequently adjusted based on the antimicrobial susceptibility of the isolates. The total duration of intravenous and subsequent oral treatment is usually 10 days, occasionally extended up to 14 days in cases with slow responses.

2. supportive care to promote healing

- adequate hydration

- minimizing trauma to the skin

- wound care

3. reduction of discomfort and the number of complications (2-3).

Our patient presented an adequate response with a favorable evolution, with the gradual remission of the symptoms.

Despite the significant morbidity of SSSS, mortality may be low among children hospitalized for treatment. In children and adults with SSSS hospitalized in the USA between 2008 and 2012, a mortality rate for children of $0.3 \%$ was reported, respectively, after the revision of the data, for adults of $4 \%$, being higher probably due to the frequent presence of severe comorbidities (5-6). SSSS recurrence is rare, reported by Davidson $\mathrm{J}$ et al. in 2017 at a newborn with extremely low birth weight.

Prevention of the disease involves minimizing the transmission risk of Staphylococcus aureus strains producing ET to young childrens (for example in newborn nurseries and intensive care units) and others at risk. Standard precautions include hand hygiene, trimming nails, adherence to precautionary protocols in hospitals, but also contact persons precautions for the entire duration of illness for the care of patients with SSSS - recommended by the Centers for Disease Control and Prevention in the United States (1-2,22). In the aforementioned outbreaks, health workers and caregivers were screened to detect the source of SSSS outbreaks (18-19).

\section{CONCLUSIONS}

SSSS is a rare disease, encountered especially in newborns and early childhood. The primary site of infection is usually the head and neck region, post- 
operative infections, umbilical area and diaper area. Diagnosis is usually based only on the clinical findings, an extensively erythematous rash respecting the mucosa. The therapy consists of antistaphylococcic penicillins and prevention of the risk of dehydration and bacterial superinfection.

\section{REFERENCES}

1. McMahon P. Staphylococcal scalded skin syndrome. In: UpToDate, Post, TW (Ed), UpToDate, Waltham, MA, 2020.

2. Mishra AK, Yadav P, Mishra A. A systemic review on staphylococcal scalded skin syndrome (SSSS): A rare and critical disease of neonates. Open Microbiol J 2016;10:150-9.

3. Leung AKC, Barankin B, Leong KF. Staphylococcal-scalded skin syndrome: Evaluation, diagnosis, and management. World J Pediatr 2018:14:116.

4. Lamand V, Dauwalder O, Tristan A et al. Epidemiological data of staphylococcal scalded skin syndrome in France from 1997 to 2007 and microbiological characteristics of Staphylococcus aureus associated strains. Clin Microbiol Infect. 2012;18(12):E514.

5. Staiman A, Hsu DY, Silverberg JI. Epidemiology of staphylococcal scalded skin syndrome in US children. British Journal of Dermatology 2018;178:704-708.

6. Staiman A, Hsu DY, Silverberg JI. Epidemiology of staphylococcal scalded skin syndrome in US adults. J Am Acad Dermatol. 2018; 79(4):774.

7. Arora P, Kalra VK, Rane S et al. Staphylococcal scalded skin syndrome in a preterm newborn presenting within first $24 \mathrm{~h}$ of life. BMJ Case Rep. 2011 Dec 21;2011:bcr0820114733.

8. Davidson J, Polly S, Hayes PJ et al. Recurrent Staphylococcal Scalded Skin Syndrome in an Extremely Low-Birth-Weight Neonate. Am J Perinatol Rep 2017;7:e134-e137.

9. Andreas H, Rosmari H, Adenilde $\mathrm{S}$ et al. Staphylococcal scalded skin syndrome in a premature newborn caused by methicillin-resistant Staphylococcus aureus: Case report. Sao Paulo Med J. 2015; 133(5):450-3.

10. Kouakou K, Dainguy ME, Kassi K. Staphylococcal Scalded Skin Syndrome in Neonate. Case Rep Dermatol Med. 2015;2015:901968.

11. Girish GM, Neeraj K, Kanwaljeet SH. Staphylococcal scalded skin syndrome: A pediatric dermatology case report. SAGE Open Medical Case Reports 2018;6:1-3.

\section{Informed consent}

Written consent was obtained from parents for the publication of this case report and any accompanying image.

Conflict of interest: none declared Financial support: none declared
12. Singh SN, Tahazzul M, Singh A, Chandra S. Varicella infection in a neonate with subsequent staphylococcal scalded skin syndrome and fatal shock. BMJ Case Rep. 2012.

13. Oliveira ARS, Aires S, Faria C et al. Staphylococcal scalded skin syndrome BMJ Case Rep. 2013.

14. Grama A, Mărginean OC, Melit LE et al. Staphylococcal Scalded Skin Syndrome in Child. A Case Report and a Review from Literature. The Journal of Critical Care Medicine 2016;2(4):192-197.

15. Haggerty J, Grimaldo F. A Desquamating Skin Rash in a Pediatric Patient. Clin Pract Cases Emerg Med. 2019;3(2):112-114.

16. Aydin D, Alsbjørn B. Severe case of staphylococcal scalded skin syndrome in a 5-year-old child - case report. Clinical Case Reports 2016;4(4):416-419.

17. Juan SU, Ji LI, Haiyan LUO et al. Demographic and clinical characteristics and risk factors for Staphylococcal scalded skin syndrome in Hunan [J]. Journal of Central South University. Medical Science, 2016;41(4): 417-421.

18. Paranthaman K, Bentley A, Milne LM et al. Nosocomial outbreak of staphyloccocal scalded skin syndrome in neonates in England, December 2012 to March 2013. Euro Surveill. 2014;19(33):1-7.

19. Pimentel de Araujo F, Tinelli M, Battisti A et al. An outbreak of skin infections in neonates due to a Staphylococcus aureus strain producing the exfoliative toxin A. Infection. 2018;46(1):49.

20. Mishra AK, Yadav P, Mishra A. A Systemic Review on Staphylococcal Scalded Skin Syndrome (SSSS): A Rare and Critical Disease of Neonates. Open Microbiol J. 2016 Aug 31;10:150-9.

21. Handler MZ, Schwartz RA. Staphylococcal scalded skin syndrome: Diagnosis and management in children and adults. J Eur Acad Dermatol Venereol. 2014;28(11):1418.

22. https://www.cdc.gov/infectioncontrol/guidelines/isolation/appendix/ type-duration-precautions.html. 